\title{
Identifying Intentional Ambiguity
}

It is widely acknowledged that certain genres in ancient Near Eastern literature including the Hebrew Bible are characterized by intense ambiguity. In particular, divination, Wisdom literature and erotic poetry thrive on a special type of ambiguity - "double-edged words" - in which a single graphic or phonetic sequence is employed to convey a message and its precise opposite, at one and the same time.

However, it is often difficult to demonstrate that a specific case of "doubleedged wording" is in fact intentional rather than a product of an eager reader's over-interpretation. The proposed paper offers three criteria for identifying intentionality in the formulation of ambiguous texts, based on examples from Biblical and other ancient Near Eastern divinatory, Wisdom and poetic texts: (1) Ungrammaticality: Sometimes an author is forced to use an ungrammatical form in order to preserve two opposite meanings. This happens when smoothing the grammar would have been achieved only at the price of losing the ambiguity; (2) Multiple representation: At times the same exact ambiguity is evidenced in identical contexts, but in different words and by means of different sentencestructures (occasionally even in different languages, e.g., Hebrew and Aramaic); when it can be demonstrated that coincidence is highly unlikely, the argument for intentional crafting is strong; (3) Straussian "Art of Writing": When the author addresses an issue that was demonstrably contentious (from the author's perspective), potentially-subversive formulations are particularly suspect.

The intersection of two or three of these criteria in a single text strongly suggests intentionality. 